\title{
Preditores da qualidade de vida e de suporte social percebido em pessoas com doença mental crónica: Estudo preliminar
}

\section{Predictors of quality of life and perceived social support in people with chronic mental disorders: Preliminary study}

\author{
Catarina Marques Ribeiro (1) \\ Rita Vanessa Alexandre Salvador (2) \\ Paula Saraiva Carvalho (3) \\ (1) Centro Municipal de Cultura e Desenvolvimento de Idanha-a-Nova (Projeto Afirma-te), Portugal \\ (2) Centro Social e Paroquial de Santa Marinha de Avanca (Projeto ESTA Integra E6G - Programa Escolhas), Portugal \\ (3) Departamento de Psicologia e Educação, Universidade da Beira Interior, Covilhã, Portugal
}

Recebido: 06/11/2018; Revisto: 21/12/2018; Aceite: 06/02/2019.

https://doi.org/10.31211/rpics.2019.5.1.100

\begin{abstract}
Resumo
Objetivos: As políticas de Saúde Mental privilegiam as práticas que incentivem a desinstitucionalização; contudo muitas pessoas com doença mental crónica permanecem institucionalizadas, competindo às instituições de saúde contribuir para melhorar a sua Qualidade de Vida e a perceção de Suporte Social. Este estudo pretende caracterizar uma amostra de pessoas com doença mental crónica institucionalizadas e identificar as variáveis preditoras da Qualidade de Vida e de Suporte Social Percebido. Métodos: Neste estudo de design transversal e descritivo-correlacional, participaram 60 mulheres institucionalizadas, com diagnóstico de Esquizofrenia ou Perturbação Bipolar. Utilizou-se um Questionário Sociodemográfico, o Questionário Breve de Avaliação da Qualidade de Vida (Vaz Serra et al., 2006) e a Escala de Satisfação com Suporte Social (Pais-Ribeiro, 1999). Resultados: As análises de regressão múltipla efetuadas demonstraram que a Perceção de Felicidade é preditora da Qualidade de Vida e do Suporte Social Percebido; a Perceção do Estado de Saúde é preditora da Qualidade de Vida e a Satisfação com a Comunidade é preditora do Suporte Social Percebido. Conclusões: Ao nível das principais conclusões e como implicações para a prática clínica, destaca-se a importância que um maior sentimento de pertença e de satisfação com a comunidade têm na Qualidade de Vida e no Suporte Social Percebido.
\end{abstract}

Palavras-Chave: Doença mental; Institucionalização; Qualidade de vida; Suporte social percebido.

\section{DI\&D | ISMT}

rpics@ismt.pt

https://rpics.ismt.pt
Publicação em Acesso Aberto

(02019. O(s) Autor(es). Este é um artigo de acesso aberto distribuído sob a Licença Creative Commons Attribution, que permite uso, distribuição e reprodução sem restrições em qualquer meio, desde que o trabalho original seja devidamente citado.
Paula Saraiva Carvalho

Departamento de Psicologia e Educação.

Universidade da Beira Interior

Estrada do Sineiro, s/n.

6200-209, Covilhã, Portugal

Tel. 275329161 | e-mail: psc@ubi.pt 


\section{Abstract}

Aims: Today's new trend is towards the deinstitutionalization of people with mental illness; however, some of them are not capable of living in the community. This incapacity implies that health institutions must improve their Quality of Life and perception of Social Support. This study aimed to characterize a sample of institutionalized people with chronic mental disorders and identify predictor variables of Quality of Life and Perceived Social Support. Method: Sixty institutionalized women, with a diagnosis of Schizophrenia or Bipolar Disorder, participated in this cross-sectional and descriptivecorrelational study. Information was assessed by a Sociodemographic Questionnaire, the World Health Organization Quality of Life - Bref Questionnaire (Vaz Serra et al., 2006), and the Social Support Satisfaction Scale (Pais-Ribeiro, 1999). Results: Multiple linear regression analyses provided evidence that Happiness Perception is a Quality of Life and a Perceived Social Support predictor; Health Condition Perception revealed to be a Quality of Life predictor and Satisfaction with Community is a Perceived Social Support predictor. Conclusions: As main conclusions of this study and implications for practice, it is highlighted the importance that a greater sense of belonging and satisfaction with the community has on the Quality of Life and Perceived Social Support.
\end{abstract}

Keywords: Mental Disorders; Institutionalization; Quality of life; Perceived social support.

\title{
Introdução
}

Alguns estudos epidemiológicos referem como principal causa de incapacidade e morbilidade nas sociedades atuais as perturbações psiquiátricas e os problemas de Saúde Mental. Das dez principais causas de incapacidade, cinco são do foro psiquiátrico (Caldas de Almeida \& Xavier, 2013). As doenças mentais crónicas, como a Esquizofrenia e a Perturbação Bipolar, são doenças que provocam uma grande severidade a nível sintomático, gerando perdas funcionais em diversas áreas da vida da pessoa e causando potenciais prejuízos em termos pessoais, familiares e socioprofissionais, com impacto na sua Qualidade de Vida (QV) (Figueiredo, Souza, Dell'Ágilo, \& Argimon, 2009).

A QV é um conceito multidimensional e subjetivo, que incorpora tanto os aspetos existenciais (e.g., sucesso pessoal relativo ao alcançar de determinados objetivos, estados ou condições desejáveis) como o sentido de bem-estar e de satisfação experienciado nas diversas áreas da vida (Canavarro, 2010). Constitui um precioso meio de avaliação do impacto das doenças na vida da pessoa com doença mental crónica, ao inserir um componente subjetivo que abrange as perceções da pessoa sobre si próprio, sobre os prejuízos que a doença lhe provoca e sobre o apoio social que julga receber ou considera essencial ou útil obter, tudo isto dentro dos seus valores pessoais, da cultura e do sistema de valores onde se insere. A QV abrange, em si mesmo, domínios como o da saúde física, psicológica e social, nomeadamente sobre os seus relacionamentos (Souza \& Coutinho, 2006). Muitos autores, principalmente no início das investigações acerca da QV, definiam esta como sinónimo de saúde (Pedroso \& Pilatti, 2010). Com o passar do tempo e os desenvolvimentos em diversas áreas, estes dois conceitos não são mais vistos como sinónimos, mas sim como contribuidores mútuos, ou seja, a saúde é um domínio da QV. No entanto, ter QV é também necessário para se ter uma boa saúde (Pedroso \& Pilatti, 2010). A perceção de QV é usualmente utilizada como medida de resultados em saúde, permitindo o controlo sintomatológico, auxiliando na compreensão do impacto das doenças, do apoio assistencial recebido e a avaliação da eficácia de determinados programas terapêuticos e de reabilitação psicossocial (Souza \& Coutinho, 2006; Teixeira, 2005). 
Por sua vez, o Suporte Social Percebido (SSP) diz respeito às funções desempenhadas por grupos ou pessoas significativas para um indivíduo em determinadas situações da vida, sendo por isso capaz de proteger e promover a saúde dos sujeitos. Estas pessoas podem ser familiares, amigos, vizinhos e outros, designando-se no conjunto por rede de suporte social(Antunes \& Fontaine, 2010).

De acordo com Ferreira e Guerra (2014) verifica-se uma relação entre a felicidade e o SSP com a QV, sendo a felicidade e o SSP variáveis que predizem a adaptação física e mental dos indivíduos. As perceções do estado de saúde, a satisfação com a vida e o SSP correlacionam-se entre si e com as emoções positivas, como a felicidade (Cloninger \& Zohar, 2011). Assim, a felicidade é um aspeto que favorece a forma como o sujeito se vê a si próprio e aos outros, o que pode resultar numa sensação de maior satisfação com as situações do dia-a-dia e com os relacionamentos com os pares, contribuindo também para a melhoria das suas capacidades de resolução de conflitos (Passareli \& Silva, 2007). Pessoas felizes são mais sociais e geralmente apresentam relações afetivas mais duradouras, são mais extrovertidas e afáveis (Quartilho, 2010).

Em doentes institucionalizados, a literatura evidencia que a QV é, geralmente, mais baixa comparativamente a pessoas com doença residentes na comunidade. Além das necessidades adicionais relacionadas com a manutenção do tratamento, muitas vezes estas pessoas com doença mental crónica são submetidas a formas de preconceito e estigma social, o que, invariavelmente, se repercute na sua QV e na sua perceção de Suporte Social (SS; Souza \& Coutinho, 2006). Na literatura é referido que a participação em programas de reabilitação psicossocial tem um efeito positivo na perceção de QV e de SSP dos indivíduos institucionalizados (Violante, 2012).

Neste sentido, apesar das políticas de Saúde Mental atuais privilegiarem as práticas que incentivem a desinstitucionalização, a avaliação da QV e do SSP em pessoas com doença mental crónica institucionalizadas continua a ser determinante pois permite, entre outras, avaliar e intervir naquilo que se pode melhorar/alterar nas instituições, de forma a contribuir para a promoção da satisfação e sucesso nos vários domínios da sua vida. Além disso, a avaliação do SSP promove o desenvolvimento de estratégias de reabilitação que contribuem para o aumento da autonomia, da realização pessoal, da felicidade e possibilitam a participação das pessoas com doença mental crónica na comunidade onde estão inseridas, usufruindo de atividades facilitadoras da sua integração social e da criação de redes sociais de suporte (Lara \& Monteiro, 2014).

Assim, este estudo tem como objetivo identificar as variáveis preditoras da QV e do SSP em mulheres com diagnóstico de esquizofrenia ou perturbação bipolar institucionalizadas em três instituições de saúde da região centro e norte de Portugal Continental.

\section{Método}

\section{Amostra}

A amostra deste estudo foi não probabilística e por conveniência, sendo constituída por 60 mulheres com diagnóstico de esquizofrenia $(n=48)$ ou perturbação bipolar $(n=12)$ institucionalizadas em estabelecimentos de saúde vocacionados para a prestação de cuidados em saúde mental. A recolha de dados foi feita de forma direta, através de autorrelato escrito, sendo o design do estudo do tipo transversal e descritivo-correlacional. 
Foram cumpridos os critérios de inclusão e exclusão previamente definidos: a) as participantes teriam de estar internadas nos estabelecimentos de saúde; b) teriam de estar diagnosticadas com esquizofrenia ou com perturbação bipolar; c) teriam de saber ler e escrever; d) ter idade superior a 18 anos e igual ou inferior a 65 anos; e) estar internadas por um período igual ou superior a 3 meses. Como critério de exclusão deliberou-se a não-presença de comorbilidades no diagnóstico.

\section{Medidas}

Aplicou-se um protocolo de investigação constituído por:

1) Um Questionário Sociodemográfico construído para este estudo e que incluiu questões sociodemográficas, clínicas e sociorrelacionais. Também para a caracterização da amostra foram acrescentados dois itens no questionário focados na avaliação da perceção de felicidade e na avaliação da perceção acerca do estado de saúde. O primeiro item era respondido através de uma questão dicotómica ("sim" ou "não") e o segundo item era respondido numa escala de tipo Likert de cinco posições, em que 1 equivalia a "péssima" e 5 equivalia a "ótima".

2) Questionário Breve de Avaliação da Qualidade de Vida [WHOQOL-BREF; World Health Organization (1997); traduzido e adaptado para a população portuguesa por Vaz Serra et al. (2006)]. O WHOQOL-BREF é uma medida multidimensional que avalia subjetivamente a perceção com a QV sendo composta por 26 itens (Canavarro et al., 2007). Na amostra do presente estudo apresentou um valor de alfa de Cronbach de 0,88 .

3) Escala de Satisfação com o Suporte Social[ESSS; Pais-Ribeiro (1999)]. A ESSS é constituída por 15 itens, de resposta tipo Likert com cinco posições. Avalia o grau de satisfação com o SS relativamente à sua família, amigos, intimidade e atividades sociais. Na amostra do presente estudo apresentou um valor de alfa de Cronbach de 0,70 .

\section{Procedimento e estratégia analítica}

O estudo cumpriu todos os princípios éticos formais para a colheita de dados. Durante a recolha de dadose no cumprimento dos princípios éticos foi assegurada a participação informada, a garantia da confidencialidade e da participação voluntária. O estudo foi aprovado pela Comissão de Ética da instituição de saúde (Ata n. ${ }^{\circ}$ 12/2014) onde foi realizada a recolha de dados, que decorreu entre janeiro e maio de 2015.

Antes da aplicação de cada protocolo era explicado a cada participante a natureza do estudo, bem como os seus objetivos, sendo formalizada a sua participação por meio da assinatura do Consentimento Informado. O protocolo assumiu a forma de autorrelato escrito e heteroadministrado pelas investigadoras. A análise estatística dos dados obtidos foi efetuada com recurso ao Statistical Package for Social Science (SPSS) versão 21.0. Para a caracterização da amostra em termos de variáveis sociodemográficas, clínicas e sociorrelacionais recorremos a estatísticas resumo (frequências absolutas e relativas, médias e desviospadrões). Para dar resposta ao objetivo, realizaram-se análises de Regressão Linear Múltipla simultânea, utilizando o método Stepwise, tendo-se confirmado os pressupostos para a sua utilização (normalidade, homocedasticidade e multicolinearidade) de acordo com as indicações de Aguiar (2007) e de Pestana e Gageiro (2008). 


\section{Resultados}

\section{Caracterização da amostra}

Relativamente à caracterização da amostra nos domínios considerados, trata-se de uma amostra de mulheres com média de idade de 50 anos ( $D P=9,14$ anos), variando entre os 31 e os 65 anos. Em termos de estado civil, $65 \%$ das participantes eram solteiras e $21,7 \%$ eram divorciadas, sendo que a maioria não tinha filhos (63,3\%). No estatuto socioeconómico verificou-se que $40 \%$ das mulheres o consideravam baixo. No que concerne ao nível de escolaridade, observou-se uma grande heterogeneidade, onde 23,3\% referiu ter o $1 .^{\circ}$ ciclo, $21,7 \%$ o $3 .^{\circ}$ ciclo e $20 \%$ o ensino secundário.

Em relação às variáveis clínicas, quanto ao tempo de diagnóstico, verificou-se uma média de 22 anos ( $D P$ $=11,56$ anos), sendo que este variou entre o 1 ano e os 47 anos.

No que respeita ao tempo de institucionalização, a média situou-se nos 8 anos e meio (100,82 meses), com o mínimo de 4 meses de internamento e no máximo 324 meses (correspondendo a 27 anos).

Quanto às variáveis sociorrelacionais, avaliou-se a frequência de visitas sendo que $35 \%$ das participantes afirmou receber visitas mensalmente, $28,3 \%$ semanalmente e $23,3 \%$ apenas anualmente. Mais de metade das participantes $(86,7 \%)$ referiu que as visitas eram de familiares. A maioria das participantes saía frequentemente da instituição $(66,7 \%)$, participava em atividades de convívio $(88,3 \%)$ e em atividades religiosas $(83,3 \%)$ e mencionava estar satisfeita com a comunidade onde estava inserida (88,3\%). Por fim, considerou-se relevante perceber qual a perceção sobre a sua felicidade e sobre a sua saúde em geral. Em relação à primeira, a amostra esteve quase igualmente distribuída, ou seja, $51,7 \%$ das respondentes afirmou não se sentir feliz e os restantes (48,3\%) disseram sentir-se felizes. Relativamente à perceção do estado de saúde geral, a pergunta subdividiu-se em cinco categorias, sendo que a maioria (51,7\%) considerou ter uma saúde razoável, 20\% afirmou ser má e 15\% disse ter uma saúde boa.

\section{Preditores da qualidade de vida e do suporte social percebido}

Em relação à análise dos preditores de QV nesta amostra, como podemos observar na Tabela 1, os resultados demonstraram que a perceção de felicidade $[\beta=0,38, t(57)=3,17 ; p<0,001]$ e a perceção do estado de saúde geral $[\beta=0,27, t(57)=2,25 ; p=0,02]$ foram os dois preditores significativos da QV neste estudo $[F(2,57)=11,89 ; p<0,00]$.

Todas as outras variáveis inseridas simultaneamente (Idade, Estado Civil, Estatuto Socioeconómico, Escolaridade, Tempo de Diagnóstico, Tempo de Institucionalização, Frequência de Visitas, Participação em Atividades de Convívio, Satisfação com a Comunidade, Realização da Higiene Pessoal Sozinha e Passar muito Tempo Sozinha) não contribuíram de forma significativa para o modelo.

A perceção de felicidade e a perceção do estado de saúde geral explicaram, em conjunto, $29,4 \%$ da variabilidade da QV. A perceção de felicidade registou um valor de beta superior ao da variável perceção do estado de saúde geral (Tabela 1).

Em relação à identificação de fatores preditores do SSP, como podemos observar na Tabela 2, a análise permitiu identificar a satisfação com a comunidade $[\beta=0,39, t(57)=3,50 ; p<0,001]$ e a perceção de felicidade $[\beta=0,29, t(57)=2,55 ; p=0,01]$ como preditores significativos da satisfação com o SSP [ $F(2$, 
$57)=10,92 ; p<0,001]$. A comparação entre os coeficientes beta permitiu verificar que a satisfação com a comunidade teve mais influência do que a perceção de felicidade na satisfação com o SSP (Tabela 2). Todas as outras variáveis, inseridas em simultâneo no modelo de regressão (Idade, Estado Civil, Estatuto Socioeconómico, Escolaridade, Número de Filhos, Tempo de Institucionalização, Frequência de Visitas, Saídas da Instituição, Participação em Atividades de Convívio, Participação em Atividades Religiosas, Perceção do Estado de Saúde Geral e Passar muito Tempo Sozinha), não contribuíram de forma significativa para o modelo. Neste modelo, $25,2 \%$ da satisfação com o SSP foi explicada com as variáveis de satisfação com a comunidade e de perceção de felicidade.

\section{Tabela 1}

Preditores de Qualidade de Vida em Pessoas com Doença Mental Crónica Institucionalizadas: Regressão Linear Múltipla

Qualidade de vida

\begin{tabular}{lccccccc}
\cline { 2 - 7 } Variáveis & $B$ & $E P$ & $\beta$ & $t$ & $p$ & $R^{2}$ & $F(g l) ; p$ \\
\hline (Constante) & 37,53 & 4,89 & & 7,67 & $<0,001$ & & \\
Perceção de Felicidade & 14,46 & 4,55 & 0,38 & 3,17 & $<0,001$ & 0,29 & $11,89(2) ;<0,001$ \\
Perceção do Estado de Saúde Geral & 5,47 & 2,42 & 0,27 & 2,25 & 0,02 & & $11,89(57) ;<0,001$ \\
\hline
\end{tabular}

Nota. $N=60 ; B=$ Coeficiente não-padronizado de regressão; $E P=$ Erro Padrão; $\beta=$ Coeficiente beta padronizado de regressão; $t=$ teste $t ; p$ = valor da significância estatística; $R^{2}=$ Coeficiente de determinação.

\section{Tabela 2}

Preditores de Suporte Social Percebido em Pessoas com Doença Mental Crónica Institucionalizadas: Regressão Linear Múltipla

\begin{tabular}{lccccccc}
\hline & \multicolumn{9}{c}{ Suporte Social Percebido } \\
\cline { 2 - 8 } Variáveis & $B$ & $E P$ & $\beta$ & $t$ & $p$ & $R^{2}$ & $F(g)) ; p$ \\
\hline (Constante) & 38,14 & 2,87 & & 2,87 & $<0,001$ \\
Satisfação com a Comunidade & 10,63 & 3,03 & 0,39 & 3,03 & $<0,001$ & 0,25 & $10,92(2) ;<0,001$ \\
Perceção de Felicidade & 4,98 & 1,94 & 0,29 & 1,94 & 0,01 & $10,91(57) ;<0,001$ \\
\hline
\end{tabular}

Nota. $N=60 ; B=$ Coeficiente não-padronizado de regressão; $E P=$ Erro Padrão; $\beta=$ Coeficiente beta padronizado de regressão; $t=$ teste $t ; p$ = valor da significância estatística; $R^{2}=$ Coeficiente de determinação.

\section{Discussão}

Muitos dos resultados que passamos a apresentar devem ser lidos no âmbito do enquadramento institucional do local onde a amostra foi recolhida. Estes estabelecimentos de saúde norteiam a sua intervenção e prática assistencial de acordo com alguns princípios e valores, bem como pela prática de estratégias de reabilitação que, de alguma forma, podem ter influenciado os resultados obtidos.

Relativamente à perceção da felicidade, que foi identificada como variável preditora da QV e do SSP neste estudo, podemos concluir que nesta amostra as participantes ficaram quase que equitativamente 
distribuídas entre o sentirem-se felizes (48,3\%) ou infelizes (51,7\%). Entre os principais motivos referidos para esta infelicidade surgiram motivos de cariz pessoal e relacional, relacionados com a doença e com o facto de esta as ter privado de viverem nas suas casas, junto dos familiares e amigos, a consciência da gravidade da sua doença e do motivo do internamento.

Por outro lado, as pessoas com doença mental que referiram sentirem-se felizes, atribuíam este sentimento às condições do local onde estavam institucionalizadas, nomeadamente o sentimento de segurança proporcionado, o sentimento de utilidade e de competência que lhes era conferido nas tarefas que executavam nas instituições, mas também ao sentimento de acolhimento e de pertença à comunidade.

Apesar de, como já vimos, felicidade não ser sinónimo de QV nem de saúde, é um constructo importante para a QV, mas também para a saúde em geral, influenciando e sendo influenciada por estas (Quartilho, 2010). As pesquisas em Psicologia que evidenciem a possibilidade do aumento da felicidade e o modo como fazê-lo são ainda muito escassas, no entanto, já existem estudos que testam algumas hipóteses (e.g., Park \& Peterson, 2009), como o incremento das possibilidades de escolha e de controlo de vários aspetos na vida destas pessoas, algo que é tido como relevante também na nossa amostra (visível através de algumas respostas dadas). Ou seja, quando as pessoas com doença mental crónica conseguem atingir um maior nível de autonomia, tendem a aumentar a sua satisfação com a vida e consequentemente a sua perceção de felicidade.

Vários estudos verificaram uma ligação entre perceção de apoio e a felicidade (e.g., Chan \& Lee, 2006), reforçando a importância dos fatores psicossociais na adaptação à doença mental crónica. Marques (2011) menciona que o grau de satisfação com o SSP desempenha um papel de relevo na perceção do bem-estar subjetivo, contribuindo para a redução de estados emocionais negativos.

Um outro preditor de QV encontrado foi a perceção do estado de saúde geral. Isto pode estar relacionado com a importância que a saúde tem para a perceção de uma boa QV. A QV depende das reações subjetivas do sujeito às suas vivências, à sua doença, dependendo sempre da sua experiência direta e da sua perceção das mesmas, bem como da felicidade e satisfação por si avaliados (Pereira, Teixeira, \& Santos, 2012). Neste estudo identificou-se que a maioria destas mulheres com doença mental crónica apresenta escassez de recursos económicos e percebeu-se que foi a admissão nestas instituições que lhes permitiu usufruir de acesso a cuidados de saúde, estabelecimento de contactos sociais, aquisição de competências e participação em atividades de lazer, o que contribui para a sua perceção do estado de saúde e de felicidade e, como consequência, para a perceção de uma boa QV.

As perceções do estado de saúde e a satisfação com a vida têm também uma relação estreita entre si, uma vez que a perceção de felicidade afeta e é afetada pela perceção do estado de saúde geral. De acordo com Taylor, Kemeny, Reed, Bower e Gruenewald (2000), pessoas mais otimistas (característica das pessoas felizes) têm crenças mais positivas acerca do futuro, funcionando como fator protetor contra o agravamento de determinadas doenças. Isto é explicado de acordo com mecanismos cognitivos, uma vez que, pacientes mais otimistas têm maior probabilidade de aderir a hábitos que promovam mais saúde, além de se apresentarem mais motivados e mais interessados no seu tratamento, o que, invariavelmente, contribui para uma melhor perceção do estado de saúde geral (Taylor et al., 2000).

Por fim, outro preditor do SSP que foi identificado foi a satisfação com a comunidade. De acordo com Elvas e Moniz (2010), "quanto maior o suporte social recebido, maior é a qualidade das relações sociais, a autoestima, a satisfação de vida e, consequentemente, maior é o sentimento de comunidade" (p. 453)." Além 
disso parece haver uma relação entre bem-estar pessoal e o sentimento de comunidade, sendo o SSP mediador desta relação (Elvas \& Moniz, 2010). Assim, o facto de as participantes desta amostra referirem que estão satisfeitas com a comunidade onde estão inseridas, pode indicar que estas não se sentem estigmatizadas nem socialmente excluídas devido à sua doença mental. A partilha de interesses e atividades que estas instituições proporcionam com a comunidade envolvente (e.g., projetos recreativos; saídas ao exterior; participação da comunidade nos serviços religiosos e festivos) parecem contribuir para que as pessoas com doença mental crónica se sintam integradas.

Fica evidente neste estudo a necessidade de avaliar a QV e o SSP em pessoas com doença mental crónica institucionalizadas. Os resultados obtidos vêm reforçar a importância da construção e aplicação de programas de reabilitação que englobem a perspetiva das pessoas com doença mental crónica que estão institucionalizadas, enfatizando novamente a importância de identificar fatores preditores para ajudar a determinar a eficácia comparativa de diferentes tratamentos e estratégias de reabilitação, avaliando assim o impacto dos mesmos na QV dos sujeitos. As estratégias de reabilitação psicossocial e de intervenção terapêutica implementadas nas instituições hospitalares onde decorreu este estudo parecem estar a funcionar como um estímulo positivo e a contribuir para a satisfação desta população, nomeadamente, para a perceção do seu estado de saúde geral e para a sua perceção de felicidade.

Existe uma relação entre a QV dos indivíduos e a comunidade onde se inserem e, por isso, pertencer a um grupo ou a uma comunidade aumenta as convicções das pessoas com doenças e a confiança pessoal nas suas capacidades, contribuindo assim para um possível aumento na sua QV (Ornelas, 2008).

Além disso, tanto a satisfação com a comunidade como a perceção de felicidade revelaram-se preditoras da satisfação com o SSP, devendo estes resultados ser tidos em consideração aquando o desenvolvimento de programas de intervenção e reabilitação. Desta forma, pessoas com algum tipo de doença, mas neste caso específico falamos das doenças mentais, poderão sentir-se integradas numa determinada rede social, bem como na comunidade, contribuindo, consequentemente, para diminuir os estados emocionais negativos e aumentar a eficácia das intervenções terapêuticas.

Apesar dos contributos para a Investigação e para a Prática Assistencial, este estudo apresenta também algumas limitações, nomeadamente, o facto de ser um estudo transversal, de não englobar participantes do género masculino e de não terem sido controladas algumas variáveis que podem também influenciar a perceção da QV e de SSP (e.g., perceção de estigma). De forma a poder generalizar os resultados da regressão seria necessário, também, aumentar a dimensão da amostra (Tabachnick \& Fidell, 2013).

\section{Principais implicações}

A nível das implicações teóricas futuras salientamos a necessidade de desenvolver instrumentos específicos para este tipo de população e a realização de mais estudos em Portugal no que diz respeito à investigação da QV e da satisfação com o SSP em pessoas com doença mental crónica. Relativamente às implicações para a prática clínica, destaca-se a importância que um maior sentimento de pertença e de satisfação com a comunidade têm em níveis mais elevados de QV e de SSP. Assim, é importante que as políticas de saúde continuem a incidir na promoção e estabelecimento de políticas de integração em contextos comunitários para consolidar a desinstitucionalização e possibilitar a reintegração plena destas pessoas. 
Apesar do enfoque das políticas atuais de saúde mental se centrar na desinstitucionalização, existem pessoas com doenças mentais que não conseguem ser reinseridas na comunidade. Compete por isso às instituições de saúde garantir as melhores práticas assistenciais contribuindo para melhorar a QV destas pessoas, bem como a prestação de SS, assegurando que estas possuem uma rede social que lhes permita sentirem-se integradas e não estigmatizadas.

A identificação de fatores preditores pode ajudar a determinar a eficácia comparativa de diferentes tratamentos e estratégias de reabilitação, avaliando o impacto dos mesmos na QV dos sujeitos. Do mesmo modo, a identificação de alguns preditores do SSP deverá ser tida em consideração na conceção e desenvolvimento de programas de intervenção e reabilitação. Desta forma, pretende-se que os profissionais de saúde mental desenvolvam práticas assistenciais mais ajustadas às necessidades e características das pessoas com doença mental crónica, tendo em conta a sua perceção de QV, contribuindo consequentemente para aumentar a eficácia das intervenções terapêuticas e a perceção de SS.

Conflito de interesses | Conflict of interest: nenhum | none.

Fontes de financiamento | Funding sources: nenhuma | none.

Contributos | Contributions: CMR e RVAS: Recolha de Dados; Revisão de literatura; Análise estatística; Redação e revisão do manuscrito. PSC: Coordenadora do estudo; Redação e revisão integral do artigo.

\section{Referências}

Aguiar, P. (2007). Guia prático de estatística em investigação epidemiológica: SPSS [Practical guide to statistics in epidemiological research: SPSS]. Lisboa: Climepsi Editores.

Antunes, C., \& Fontaine, A. M. (2010, fevereiro). Adaptação de uma Escala de Avaliação do Suporte Social - NOS (Network Orientation Scale) [Adaptation of a Social Support Assessment Scale - NOS (Network Orientation Scale)]. Paper presented at the VII Simpósio Nacional de Investigação em Psicologia (pp. 15-29). Braga, Portugal. Retrieved from https://repositorio-aberto.up.pt/bitstream/10216/21507/2/85418.pdf

Caldas de Almeida, J. M., \& Xavier, M. (2013). Estudo epidemiológico nacional de saúde mental: ${ }^{\circ}$ relatório [National epidemiological study of mental health: 1st report]. Retrieved from http://www.fcm.unl.pt/main/alldoc/galeria_imagens/Relatorio_Estudo_Saude-Mental_2.pdf

Canavarro, M. C. (2010). Qualidade de vida: Significados e níveis de análise [Quality of life: Meanings and levels of analysis]. In M. C. Canavarro \& A. Vaz-Serra (Eds.), Qualidade de vida e saúde: Uma abordagem na perspectiva da Organização Mundial de Saúde [Quality of life and health: An approach from the perspective of the World Health Organization] (pp. 3-22). Lisboa: Fundação Calouste Gulbenkian.

Canavarro, M. C., Simões, M. R., Vaz-Serra, A., Pereira, M., Rijo, D., Quartilho, M. J., ... Carona, C. (2007). Instrumento de avaliação da qualidade de vida da Organização Mundial de Saúde: WHOQOL-Bref [World Health Organization quality of life assessment tool: WHOQOL-Bref]. In M. Simões, C. Machado, M. Gonçalves, \& L. Almeida (Eds.), Avaliação psicológica: Instrumentos validados para a população portuguesa [Psychological evaluation: Instruments validated for the Portuguese population] (Vol. III, pp. 77-100). Coimbra: Quarteto Editora.

Chan, Y. K., \& Lee, R. P. L. (2006). Network size, social support and happiness in later life: A comparative study of Beijing and Hong Kong. Journal of Happiness Studies, 71), 87-112. https://doi.org/10.1007/s10902-0051915-1

Cloninger, C. R., \& Zohar, A. H. (2011). Personality and the perception of health and happiness. Journal of Affective Disorders, 128(1-2), 24-32. https://doi.org/10.1016/j.jad.2010.06.012

Elvas, S., \& Moniz, M. J. V. (2010). Sentimento de comunidade, qualidade e satisfação de vida [Feeling of community, quality and life satisfaction]. Análise Psicológica, 28(3), 451-464. Retrieved from http://www.scielo.mec.pt/pdf/aps/v28n3/v28n3a06.pdf 
Ferreira, M., \& Guerra, M. P. (2014). Adaptação à lesão vertebro-medular [Adjustment to spinal cord injury]. Psicologia, Saúde \& Doenças, 15(2), 380-395. Retrieved from http://www.scielo.mec.pt/pdf/psd/v15n2/v15n2a05.pdf

Figueiredo, A., L., Souza, L., Dell'Ágilo Jr., J. C., \& Argimon, I. I. L. (2009). O uso de psicoeducação no tratamento do transtorno bipolar [Use of psychoeducation in the treatment of bipolar disorder]. Revista Brasileira de Terapia Comportamental e Cognitiva, 11(1), 15-24. Retrieved from http://www.usp.br/rbtcc/index.php/RBTCC/article/view/412/306

Lara, G. A., \& Monteiro, J. K. (2014). Reabilitação psicossocial de pessoas com transtornos psicóticos: Atuação dos psicólogos nos CAPS de Santa Catarina [Psychosocial rehabilitation of individuals with psychotic disorders: The role of psychologists in CAPS of Santa Catarina state]. Contextos Clínicos, 71), 49-61. https://doi.org/10.4013/ctc.2014.71.05

Marques, V. F. B. (2011). Influência do suporte social e dos estilos de coping na perceção de bem-estar subjetivo em doentes mentais crónicos [Influence of social support and coping styles on subjective well-being perception in chronic mentally ill patients] (Master's thesis, Instituto Superior Miguel Torga, Coimbra). Retrieved from https://core.ac.uk/download/pdf/49288008.pdf

Ornelas, J. (2008). Psicologia comunitária[Community psychology]. Lisboa: Fim de Século.

Pais-Ribeiro, J. L. (1999). Escala de Satisfação com o Suporte Social (ESSS) [Social Support Satisfaction Scale (ESSS)]. Análise Psicológica, 173), 547-558. Retrieved from http://www.scielo.mec.pt/scielo.php?script=sci_arttext\&pid=S0870-82311999000300010

Park, N., \& Peterson, C. (2009). Achieving and sustaining a good life. Perspectives on Psychological Science, 4(4), 422-428. https://doi.org/10.1111/j.1745-6924.2009.01149.x

Passareli, P. M., \& Silva, J. A. (2007). Psicologia positiva e o estudo do bem-estar subjetivo [Positive psychology and the subjective well-being study]. Estudos de Psicologia, 24(4), 513-517. Retrieved from http://www.scielo.br/pdf/estpsi/v24n4/v24n4a10.pdf

Pedroso, B., \& Pilatti, L. A. (2010). Avaliação de indicadores da área da saúde: A qualidade de vida e suas variantes [Indicators evaluation of the health area: Quality of life and its variants]. Revista Eletrônica da Faculdade Integrada de Itaré, 1(1), 1-9. http://www.fafit.com.br/revista/index.php/fafit/article/viewFile/2/2

Pereira, É. F., Teixeira C. S., \& Santos, A. (2012). Qualidade de vida: Abordagens, conceitos e avaliação [Quality of life: Approaches, concepts and assessment]. Revista Brasileira de Educação Física e Esporte, 26(2), 241-250. https://doi.org/10.1590/S1807-55092012000200007

Pestana, M. H., \& Gageiro, J. N. (2008). Análise de dados para Ciências Sociais: A complementaridade do SPSS [Data analysis for Social Sciences: The complementarity of SPSS] ( $5^{\text {th }}$ ed.). Lisboa: Edições Sílabo.

Quartilho, M. J. R. (2010). Qualidade de vida, felicidade, saúde, bem-estar, satisfação. Pessoas, sociedades, culturas. O que importa? [Quality of life, happiness, health, well-being, satisfaction. People, societies, cultures. What matters?]. In M. C. Canavarro \& A. Vaz-Serra (Eds.), Qualidade de vida e saúde: Uma abordagem na perspectiva da Organização Mundial de Saúde [Quality of life and health: An approach from the perspective of the World Health Organization] (pp. 55-128). Lisboa: Fundação Calouste Gulbenkian.

Souza, L. A., \& Coutinho, E. S. F. (2006). Factores associados à qualidade de vida de pacientes com esquizofrenia [Associated factors to quality of life of patients with schizophrenia]. Revista Brasileira de Psiquiatria, 28(1), 50-58. https://doi.org/10.1590/S1516-44462006000100011

Tabachnick, B. G., \& Fidell, L. S. (2013). Using multivariate statistics (6th ed.). Boston: Pearson Education.

Taylor, S. E., Kemeny, M. E., Reed, G. M., Bower, J. E., \& Gruenewald, T. L. (2000). Psychological resources, positive illusions, and health. American Psychologist, 55(1), 99-109. https://doi.org/10.1037/0003-066X.55.1.99

Teixeira, M. B. (2005). Qualidade de vida de familiares cuidadores do doente esquizofrênico [Life quality of family caregivers of schizophrenic patient]. Revista Brasileira de Enfermagem, 58(2), 171-175. https://doi.org/10.1590/S0034-71672005000200008

Vaz Serra, A., Canavarro, M. C., Simões, M. R., Pereira, M., Gameiro, S., Quartilho, M. J., ... Paredes, T. (2006). Estudos psicométricos do instrumento de avaliação da qualidade de vida da Organização Mundial de Saúde (WHOQOL-Bref) para Português de Portugal [Psychometric studies of the World Health Organization Quality of Life Assessment (WHOQOL-Bref) for Portuguese from Portugal]. Psiquiatria Clínica, 271), 41-49. Retrieved from https://estudogeral.sib.uc.pt/bitstream/10316/21539/1/2006\%20Estudos\%20psicométricos\%20do\%20W HOQOL-Bref.pdf 
Violante, C. D. F. (2012). Programas de reabilitação psicossocial em pessoas com doença mental crónica: Impacto na perceção de qualidade de vida e de suporte social [Psychosocial rehabilitation programs in people with chronic mental illness: Impact on the perception of quality of life and social support] (Master's thesis, Instituto Politécnico de Bragança, Bragança). Retrieved from http://hdl.handle.net/20.500.11960/1224

World Health Organization. (1997). WHOQOL measuring quality of life. Geneva: WHO. Retrieved from https://apps.who.int/iris/bitstream/handle/10665/63482/WHO_MSA_MNH_PSF_97.4.pdf?sequence=1\&is Allowed=y 